\section{Respostas fisiológicas da Posição Mãe- Canguru em bebês pré-termos, de baixo peso e ventilando espontaneamente}

\section{Physiological responses of the Kangaroo Mother Position in low birth weight, spontaneous ventilating premature babies}

Aline R Miltersteiner 1

Diego R Miltersteiner 2

Viviane $V$ Rech 3

Lucas Dalle Molle 4

\section{Abstract}

Objectives: to determine physiological responses such as heart rate, oxygen saturation, axillary temperature and respiratory frequency in premature babies kept in the Kangaroo Mother Position (KMP).

Methods: twenty three premature babies with stable hemodynamic conditions, in spontaneous ventilation, and no lung disease, inpatients of the Center of Neonatology of the Hospital Conceição, Porto Alegre, were assigned to the Group I (control group) and Group II (KMP). A pre-test, post-test intervention study design was used in which babies were their own controls. Data were registered in the first minute (T01) to the 30th minute (T30) and from the 30th to the 60 th minute (T60). Student's " $t$ " test was used to compare both groups.

Results: patients present a mean gestational age of 34 weeks, mean weight of $1780 \mathrm{~g}$ and median age of 264 hours of life. Increase with statistical significant difference in oxygen saturation at T30 and T60 was demonstrated, as well as heart rate at T30 and axillary temperature a T60, when comparing Group II to $I$.

Conclusions: KMP promotes improvement of physiological parameters in low weight premature children, when procedure is initiated within a period of one hour as compared to the same observation period in the incubator. KMP is possible during physiotherapeutic care.

Key words Kangaroo Mother Method, Infant, premature, Physical therapy

\section{Resumo}

Objetivo: avaliar as respostas fisiológicas - freqüência cardíaca, saturação periférica de oxigênio, temperatura axilar e freqüência respiratória - em bebês pré-termos estáveis e em ventilação espontânea, submetidos à observação na incubadora e à Posição Mãe-Canguru.

Métodos: foram estudados 23 pré-termos estáveis hemodinamicamente, em ventilação espontânea, sem doença pulmonar diagnosticada, provenientes do Centro de Neonatologia do Hospital Conceição, Porto Alegre. Os pacientes foram distribuídos em Grupo I (incubadora) e Grupo II (Posição Mãe-Canguru) para um estudo de intervenção, de amostras pareadas, sendo cada paciente controle de si mesmo. Os dados foram registrados no primeiro minuto (T01), aos 30 (T30) e aos 60 minutos (T60). Utilizouse o teste t de Student para comparação entre os grupos.

Resultados: os pacientes apresentaram uma média de idade gestacional de 34 semanas, média de peso pós-natal de $1780 \mathrm{~g}$ e mediana de 264 horas de vida. Observou-se um aumento estatisticamente significante na freqüência cardíaca em T30, na saturação de oxigênio em T30 e T60 e na temperatura axilar em T60, comparando o grupo da Posição Mãe-Canguru ao grupo controle.

Conclusões: a Posição Mãe-Canguru promoveu aumento nos valores das respostas fisiológicas estudadas em pré-termos, quando instituída por uma hora, em comparação ao mesmo período na incubadora, sendo possível sua utilização durante atendimento fisioterapêutico.

Palavras-chave Método Mãe-Canguru, Prematuro, Fisioterapia, Processos fisiológicos 


\section{Introdução}

Estudos sobre os efeitos de programas de intervenção envolvendo bebês pré-termos têm produzido evidências substanciais de que essas intervenções, tais como estimulações multimodal, proprioceptiva, tátil, vestibular, cinestésica, auditiva, visual ou rítmica apresentam efeitos favoráveis no desenvolvimento desses pacientes. ${ }^{1}$

A melhora do estado geral dos pré-termos, secundária a esses programas de intervenção, usualmente manifesta-se por meio de um melhor padrão alimentar, menor necessidade de oxigênio, menor tempo de suporte ventilatório, menor incidência de apnéia, maior ganho de peso, menor período de alimentação via sonda, menor tempo de hospitalização e melhora do desenvolvimento social.2,3

Sabe-se que, anualmente, nascem em todo o mundo, vinte milhões de bebês com baixo peso e pré-termos. Desses, $40 \%$ morrem antes de completar o primeiro ano de vida. Nos hospitais dos países em desenvolvimento há grande escassez de recursos, infra-estrutura inadequada, superlotação, altas taxas de infecções hospitalares e riscos elevados de morbimortalidade. ${ }^{4}$

Como resposta a esses problemas de atendimento ao recém-nascido (RN) pré-termo e de baixo peso, o Método Mãe-Canguru foi desenvolvido, no Instituto Materno Infantil de Bogotá, Colômbia. Essa iniciativa adotou seu nome em referência a espécie dos marsupiais, na qual os filhotes nascem antes de completar a gestação.4,5

Esse método é uma alternativa ao método tradicional, disponível e dispendioso, de tratamento dos bebês pré-termos que são assistidos e tratados em incubadoras.

Desde a implantação do método muito tem sido publicado sobre o assunto na busca de recursos que confirmem sua segurança e eficiência em pré-termos de baixo peso. Trabalhos versando sobre as respostas fisiológicas apresentam desde diminuição da frequiência de apnéias, de episódios de bradicardia e até o aumento da oxigenação. 4,6-9

No Brasil, o Ministério da Saúde trabalha na disseminação, treinamento e implementação dos centros de referência. As primeiras instituições que aplicaram essa nova tecnologia foram o Hospital Guilherme Álvaro, em Santos (1992) e o Instituto Materno Infantil de Pernambuco (IMIP) em Recife (1994). A partir disso, outros hospitais adotaram o método como rotina hospitalar, dentre eles o Hospital de Itapecerica da Serra, em São Paulo, o Hospital Geral César Cals, no Ceará, o Hospital Regional de Taguatinga, no Distrito Federal, o Hospital Univer- sitário, em Florianópolis, o Hospital de Clínicas do Paraná, entre outros. Atualmente são mais de 160 programas em andamento no país. 10

Os custos de atenção aos neonatos que recebem os cuidados do método são relativamente baixos em comparação ao método tradicional, ${ }^{11}$ por ser um programa simples, próprio para países com recursos escassos. Ainda, é um modelo eficaz, com ótima relação custo-benefício, pois incrementa a sobrevida, a qualidade de vida e evita o abandono durante a internação, freqüente nesses casos. 5,12

Uma vez que esse método não é uma realidade no Rio Grande do Sul, pretende-se por meio deste estudo despertar o interesse para a utilização do mesmo nas unidades de terapia intensiva neonatais (UTIN) a partir de seu componente "posição" e salientar a participação do profissional fisioterapeuta como integrante da equipe multidisciplinar na instituição da Posição e/ou do Método Mãe-Canguru, aprimorando as formas de tratamento e assistência fisioterapêutica em hospitais e maternidades.

O presente estudo teve como objetivo verificar as respostas fisiológicas, a partir da frequiência cardíaca (FC), saturação periférica de oxigênio (SpO2), frequiência respiratória (FR) e temperatura corporal (Tax), em bebês pré-termos assistidos tanto em incubadoras quanto submetidos à Posição MãeCanguru.

\section{Métodos}

Foi realizado um estudo de intervenção, descritivo e de amostras pareadas, na UTIN do Hospital da Criança Conceição e no Berçário do Hospital Nossa Senhora da Conceição (Grupo Hospitalar Conceição), entre o período de julho de 2000 a agosto de 2001, em Porto Alegre, RS, Brasil.

O cálculo do tamanho amostral foi realizado para um tamanho de efeito padronizado de 1, um nível de significância de 0,05 e um poder do estudo de $80 \%$.

Foi estudada uma amostra consecutiva, de 23 bebês pré-termos de ambos os sexos, assistidos em incubadora, estáveis hemodinamicamente, com peso igual ou inferior a $2000 \mathrm{~g}$, nascidos com idade gestacional entre 24 a 37 semanas e com boa capacidade de sucção e deglutição. Os critérios de elegibilidade das mães compreenderam: motivação, disciplina, disponibilidade e compromisso, ausência de problemas mentais ou lesões dermatológicas.

Foram excluídos os binômios mãe-bebê quando um ou ambos apresentassem doenças ou se o pré-termo necessitasse de ventilação mecânica.

Os dados dos bebês foram obtidos por meio de 
informações obtidas no prontuário médico e pela entrevista com a mãe, em um protocolo definido. Os pré-termos foram agrupados conforme o tipo de posição, constituindo o Grupo I, posicionados na incubadora; e Grupo II, submetidos na Posição MãeCanguru, sendo cada paciente controle de si mesmo.

As medidas do desfecho principal foram aferidas por meio da observação das seguintes respostas fisiológicas: frequiência cardíaca (em batimentos cardíacos por minuto, bpm), saturação periférica de oxigênio (em percentual), temperatura corporal (em graus Celsius) e freqüência respiratória (em movimentos respiratórios por minuto, mrpm), no primeiro (T01), aos 30 (T30) e aos 60 minutos (T60) de observação, em pré-termos em ventilação espontânea, submetidos ao decúbito elevado na incubadora e à Posição Mãe-Canguru, no período de uma hora.

Após o intervalo de uma hora da alimentação, foram aferidas as medidas para o Grupo I. A seguir, os bebês recebiam alimentação e uma hora após eram colocados na Posição Mãe-Canguru, então sendo realizadas as medidas para o Grupo II. Todas as observações foram conduzidas pela mesma pesquisadora (ARM) e no turno da tarde, de acordo com a disponibilidade das mães. O desenho do estudo encontra-se representado na Figura 1.

Figura 1

Seqüência dos procedimentos no estudo.

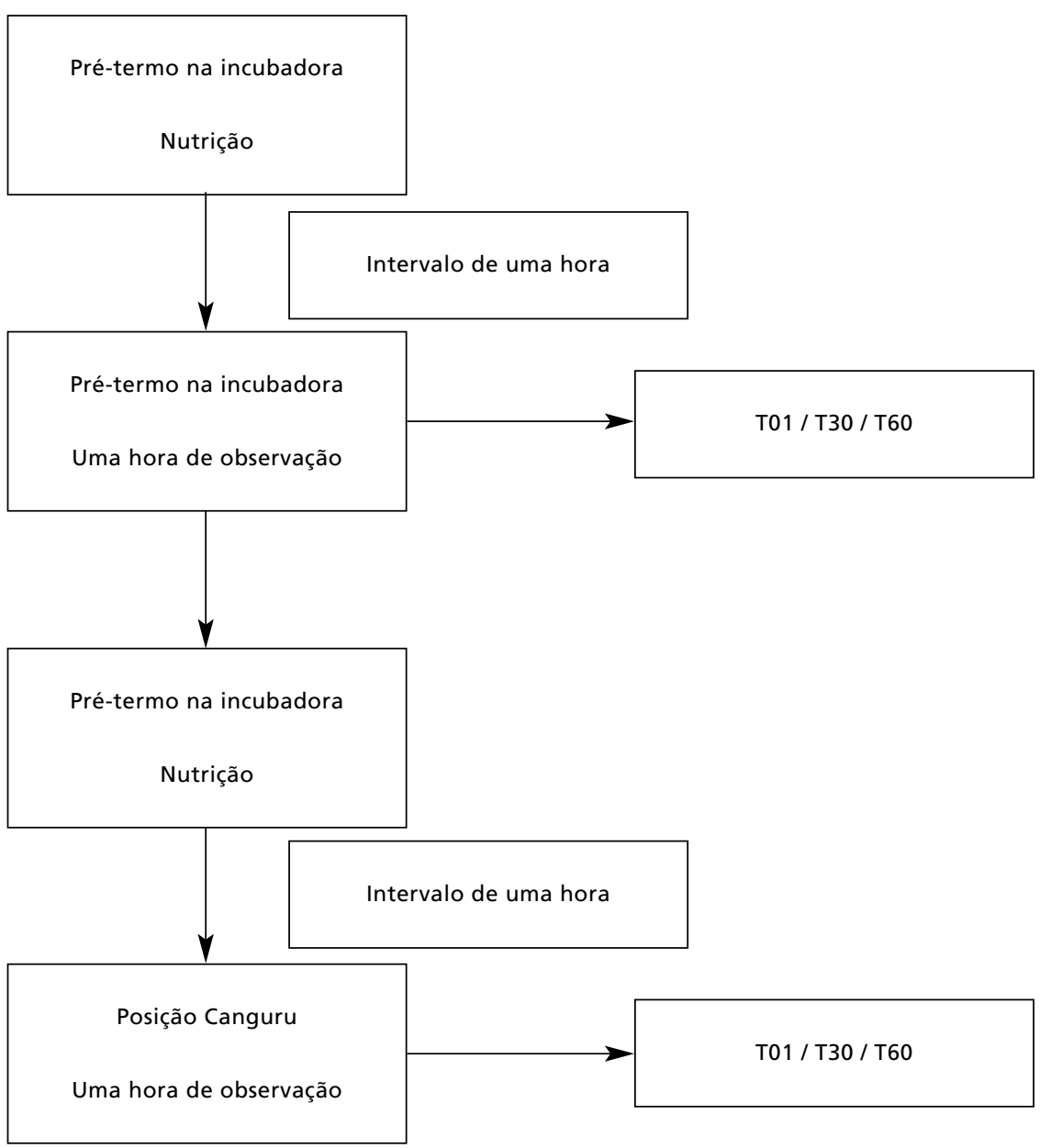


No presente trabalho, optou-se por pré-termos de baixo de peso sem doença de base, seguindo os critérios de elegibilidade estabelecidos por Charpak et al. 4

Os bebês foram observados em ambos os posicionamentos após uma hora da amamentação, devido à influência da alimentação sobre o consumo de oxigênio, uma vez que existe aumento desse consumo pós-prandialmente em crianças que receberam nutrição total oral. ${ }^{6}$ Por essa razão, não houve nenhuma forma de nutrição durante os períodos de estudo. De acordo com modelos descritos na literatura, as observações foram realizadas uma hora depois da amamentação $4,6,12$ em ambos os grupos, utilizando-se uma hora de estudo para cada grupo.

Houve a escolha pelo período de tempo de uma hora para a realização da Posição Mãe-Canguru pelas características da UTI neonatal onde foi conduzido o estudo. Além disso, já foi descrito na literatura um estudo que demonstrou alteração das respostas fisiológicas com apenas dez minutos de observação. 13

A Posição Mãe-Canguru foi realizada pela mãe, 4 no intuito de manter os resultados das respostas fisiológicas mais homogêneos, pois o mesmo posicionamento conduzido pelos pais tem demonstrado maior aumento nos valores referentes às frequiências respiratória e cardíaca, temperatura retal e cutânea, sendo relacionado a um maior consumo de oxigênio do bebê pré-termo durante a posição com o Pai Canguru, devido à condução de calor do pai ser maior que a da mãe, sem implicar em aumento da produção de calor pelo bebê. 12

\section{Definição dos grupos}

O Grupo I (incubadora) permaneceu em decúbito ventral elevado a $40^{\circ} \mathrm{C}$ por um suporte de espuma padronizado, colocado sob o suporte do colchão da incubadora, no intuito de obter-se uma posição semelhante àquela proporcionada pela Posição MãeCanguru (Figura 2).

A temperatura da incubadora (FANEM C86H, Guarulhos, SP, Brasil) foi controlada por meio do termômetro de coluna de mercúrio da mesma, permanecendo entre 28 e $30^{\circ} \mathrm{C}$, de acordo com a necessidade de cada pré-termo.

Para o Grupo II utilizou-se a Posição Mãe-Canguru, que consistiu no bebê ser colocado junto ao seio materno, em contato pele a pele, estando a mãe em uma cadeira acolchoada a $60^{\circ} \mathrm{C}$ de inclinação (Figura 3). Ele ficava seguro pelo avental vestido pela mãe, evitando-se assim a perda de calor para o meio.
Não houve amamentação durante a Posição MãeCanguru ou qualquer forma de nutrição durante o decúbito elevado. A temperatura da sala durante a realização do estudo permaneceu entre $28-30^{\circ} \mathrm{C}$, controlada a partir do termômetro (Incoterm Ltda, Porto Alegre, RS, Brasil) fixado na parede da sala da UTIN ou do Berçário.

\section{Figura 2}

Bebê pré-termo no decúbito ventral elevado.

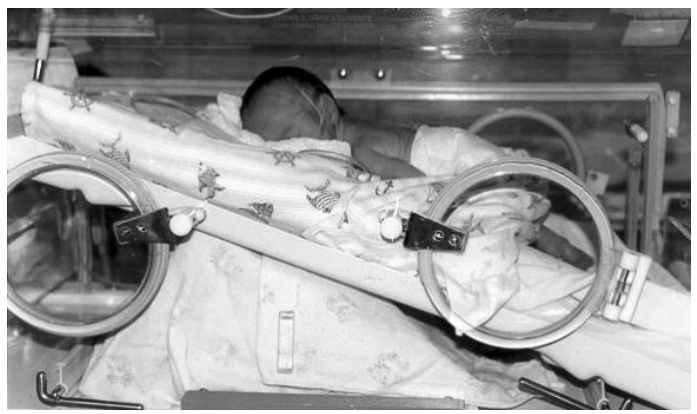

\section{Figura 3}

Dupla mãe-bebê durante a realização da Posição MãeCanguru.

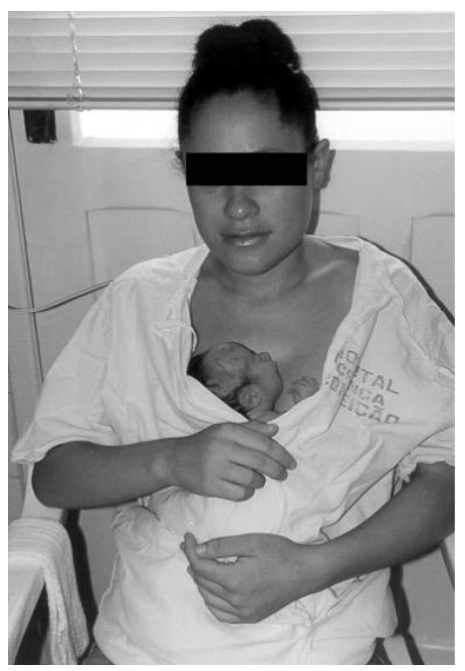




\section{Métodos de aferição}

A freqüência respiratória foi verificada a partir da observação das excursões torácicas do bebê, durante um minuto completo. Foram verificados os valores da freqüência cardíaca e da saturação periférica de oxigênio, com a utilização de oxímetro de pulso (JG Moriya 1001, JG Moriya Indústria e Comércio, São Paulo, SP, Brasil) com sensor neonatal adesivo reutilizável (JG Moriya 3026, JG Moriya Indústria e Comércio, São Paulo, SP, Brasil), colocado no pé direito do pré-termo.

A medida da temperatura foi realizada com o uso de um termômetro de coluna de mercúrio (Incoterm, Porto Alegre, RS, Brasil), colocado sobre a região axilar direita, previamente seca, por no mínimo quatro minutos, após a aferição das variáveis anteriores, diminuindo a interferência do estresse pela manipulação do bebê.

Os valores da frequiência cardíaca, da saturação de oxigênio, da temperatura corporal e da freqüência respiratória amostras pareadas, sendo considerado um valor de $\mathrm{p}$ menor do que 0,05 como estatistica- mente significante. Foi utilizado o software Statistic Package for Social Sciences (SPSS) versão 10, para os cálculos estatísticos.

Este estudo teve aprovação do Grupo de Ética em Pesquisa do Grupo Hospitalar Conceição contando com a participação do dupla mãe-bebê após a assinatura, pelas mães, do "Termo de Consentimento Livre e Esclarecido" e do "Termo de Consentimento do Registro Fotográfico para a Publicação Científica".

\section{Resultados}

A amostra estudada apresentou $65 \%$ de bebês prétermos do sexo masculino e $43 \%$ dos partos por cirurgia cesárea. A mediana de idade dos bebês na inclusão do estudo foi de 264 horas ou 11 dias de vida (intervalo interquartílico: $128-480$ horas). Trinta e cinco porcento das mães tinham idade inferior a 20 anos, $51 \%$ eram primíparas e $69 \%$ tinham o ensino fundamental incompleto. Os demais resultados das variáveis descritivas encontram-se na Tabela 1.

Tabela 1

Valores médios ( \pm dp) e amplitude das características clínicas e biológicas das crianças.

\begin{tabular}{lccc}
\hline Variáveis & $\overline{\mathbf{X}} \pm \mathbf{d p}$ & Mínimo & Máximo \\
\hline Idade gestacional ao nascer* & $34,1 \pm 1,88$ & 24 & 37 \\
Apgar no primeiro minuto & $6,48 \pm 2,02$ & 2 & 9 \\
Apgar no quinto minuto & $8,43 \pm 1,04$ & 37 & 48 \\
Comprimento ao nascer (cm) & $41,87 \pm 2,67$ & 1210 & 2100 \\
Peso ao nascer (g) & $1740 \pm 280,04$ & 1535 & 1995 \\
Peso na inclusão no estudo (g) & $1780 \pm 116,08$ & 6 \\
\hline
\end{tabular}

*Expressa em semanas de gestação.

A nutrição dos bebês constituiu-se principalmente por leite materno oferecido a partir do seio, para todos, intercalado com o leite materno por meio de copinho em $56,5 \%$. Somente $13 \%$ dos prematuros fizeram uso de fórmula complementar de nutrição concomitante ao leite materno.

Observou-se boa capacidade de deglutição e a presença do reflexo de sucção em todos os bebês.

Verificou-se aumento nos valores da freqüência cardíaca dos bebês do grupo Posição Mãe-Canguru
(Grupo II) em comparação ao mesmo período de tempo de observação do grupo incubadora (Grupo I), aos 30 minutos de observação $(p=0,001)$. Os resultados dessa resposta fisiológica referentes ao primeiro minuto (T01) e aos 60 minutos (T60) não apresentaram diferença estatística entre os dois grupos. Há semelhança da observação do T01 para os valores da saturação periférica de oxigênio, da freqüência respiratória e da temperatura axilar, quando comparados os valores das respostas fisiológicas en- 
tre os bebês observados na incubadora e quando submetidos à Posição Mãe-Canguru. Não houve diferença estatística nos tempos de observação dos valores da frequiência respiratória, quando comparados ambos os grupos, nos mesmos períodos de tempo (Tabela 2).

$\mathrm{Na}$ verificação da saturação periférica de oxigênio, observou-se um aumento nos valores do Grupo II em comparação ao Grupo I, nos mesmos períodos de tempo, à observação de T30 e T60, ( $p=0,005$ e 0,017 , respectivamente). Da mesma forma, encontrou-se aumento nos valores da temperatura axilar aos 60 minutos de observação dos bebês submetidos à Posição Mãe-Canguru em comparação ao mesmo período de tempo que foram observados no decúbito elevado na incubadora, com diferença estatística $(\mathrm{p}=0,005)$ (Tabela 2).

Tabela 2

Valores médios ( \pm dp) e amplitudes das respostas fisiológicas e níveis de significância entre os dois grupos, em cada intervalo de tempo.

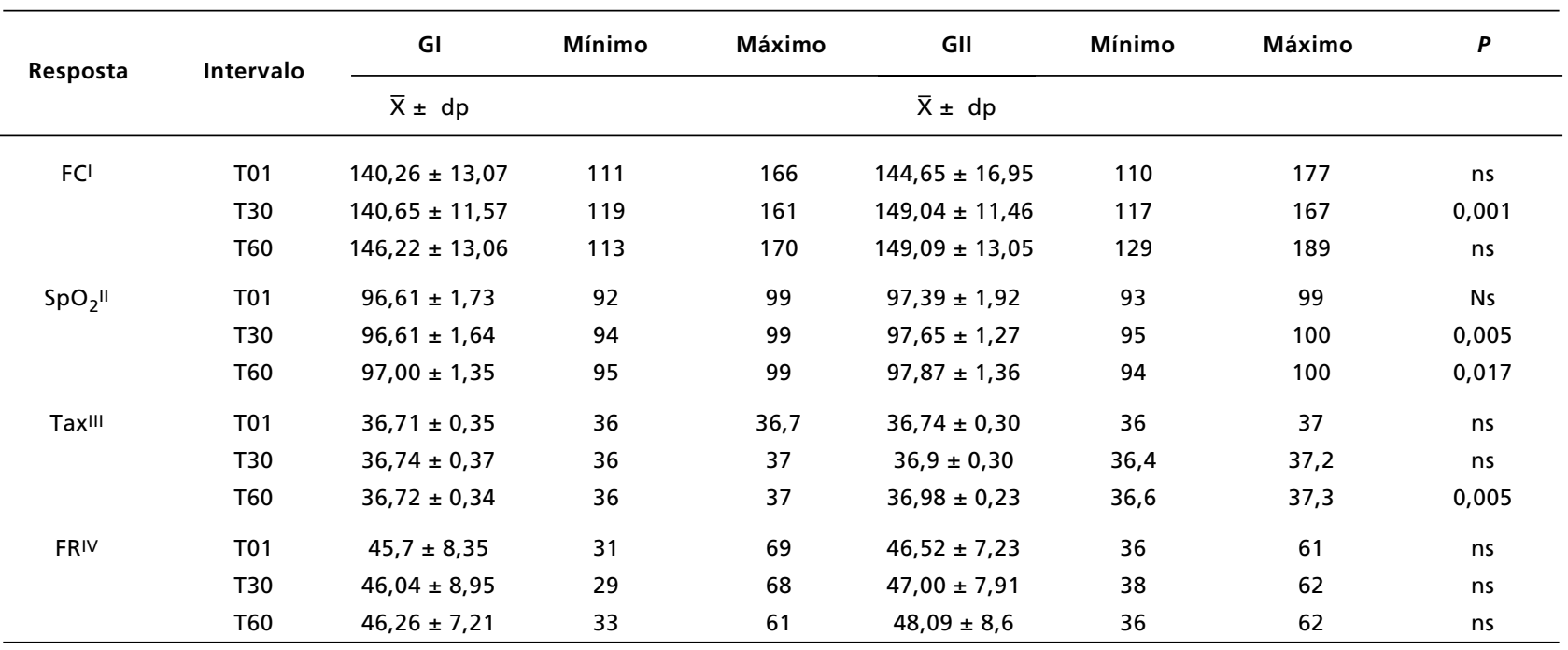

$\mathrm{P}=$ nível de significância; Mínimo = Valor mínimo; Máximo = Valor máximo; GI = Grupo incubadora; GII = Grupo Posição Mãe-Canguru; dp = desvio-padrão; ns = Não-significante; I. Freqüência cardíaca, em batimentos por minuto; II. Saturação periférica de oxigênio, em percentual; III. Temperatura axilar, em graus Celsius; IV. Freqüência respiratória, em movimentos respiratórios por minuto; * Momento de aferição: $\mathrm{T} 01-10$ minuto, $\mathrm{T} 30=30$ minutos, $\mathrm{T} 60=60$ minutos

\section{Discussão}

Diversos estudos têm sido conduzidos com o objetivo de avaliar as respostas fisiológicas, a estabilidade térmica, o ganho ponderal e a relação mãe-bebê das crianças assistidas por esse cuidado ou submetidas à Posição Mãe-Canguru.1,5-8,11,12,14

Até a data de sua conclusão esse estudo é o primeiro que avalia as respostas fisiológicas da Posição Mãe-Canguru, realizado no Brasil.

A partir dos resultados desse estudo, há intenção de promover essa posição como forma de atendimento fisioterapêutico aos pré-termos, pois sabe-se que o período total de tempo desse atendimento a esses bebês internados em unidade de tratamento intensivo é menor que nos demais casos pela fragilidade dos mesmos. 15

A observação desses prematuros, no decúbito ventral elevado, foi realizada devido aos benefícios já descritos na literatura científica, como melhora da oxigenação em bebês com disfunção respiratória, que parece residir em efeitos nas relações ventilação-perfusão, volumes e capacidades pulmonares. ${ }^{16,17}$ Sabe-se que crianças com insuficiência respiratória aguda colocadas em decúbito ventral elevado apresentam oxigenação significativamente superior àquelas em decúbito dorsal, a explicação para tal fato está na distribuição gravitacional da 
pressão pleural que é muito mais uniforme no primeiro posicionamento. ${ }^{18,19}$ Embora nenhum bebê tenha apresentado qualquer condição respiratória, esse posicionamento foi considerado seguro para ser aplicado ao grupo controle e por ser o decúbito permitido na incubadora que mais assemelhou-se à posição vertical promovida pela Posição Mãe-Canguru.

A análise dos resultados mostrou que a freqüência cardíaca, a saturação de oxigênio e a temperatura axilar dos bebês submetidos à Posição Mãe-Canguru foram mais elevadas em comparação com o mesmo período de tempo que estavam na incubadora em decúbito ventral elevado. Esses dados permitiram comparações com os achados de outros autores que estudaram o mesmo tema.6-8,14,20

O aumento da frequência cardíaca nos pré-termos submetidos à Posição Mãe-Canguru foi registrado aos 30 minutos de observação e da saturação periférica de oxigênio aos 30 e aos 60 minutos em comparação aos mesmos períodos no posicionamento em decúbito ventral elevado na incubadora, com diferença estatística significante.

Sabe-se que o consumo de oxigênio aumenta linearmente com o aumento da frequiência cardíaca. Kollef 21 analisou a relação entre consumo e saturação de oxigênio por meio da Equação de Fick, utilizada para calcular o gasto energético em pacientes, na qual observa-se que essas variáveis são diretamente proporcionais entre si. Dessa forma, constatase um aumento no saturação periférica provavelmente mediado pelo aumento da freqüência cardíaca, que por sua vez alterou-se em conseqüência do incremento da temperatura do bebê em contato pele a pele com sua mãe, sendo que essa elevação na temperatura também foi significante aos 60 minutos de observação da Posição Mãe-Canguru em comparação ao mesmo período de observação do decúbito ventral elevado na incubadora. Entretanto, essa posição está associada a maior duração do sono tranqüilo e redução da atividade, 22 o que paradoxalmente conduziria a um menor gasto metabólico. $\mathrm{O}$ aumento na frequiência cardíaca desses bebês pode estar relacionado ao aumento temporário da força contrátil do coração, influenciado pelo aumento da permeabilidade iônica da membrana celular, resultado do aumento moderado da temperatura, levando à aceleração do processo de auto-excitação do ciclo cardíaco. 23

Esse incremento na temperatura foi observado ao final de uma hora de observação dos bebês que realizaram a posição vertical. No estudo de Bauer $e t$ $a l ., 6$ verificou-se que a temperatura cutânea periférica e retal foi maior durante o contato pele a pele pro- movido pela posição canguru, realizada no período de uma hora, em pré-termos com peso inferior a $1500 \mathrm{~g}$ e ventilando espontaneamente. Da mesma forma que Bohnhorst et al. ${ }^{8}$ demonstraram aumento estatisticamente significante da temperatura retal nos pré-termos quando submetidos à Posição Mãe-Canguru, sem alteração significativa quando não estavam em contato pele a pele, sendo realizada a medida a cada duas horas, ao invés do período de uma hora. Esses achados foram compatíveis aos encontrados neste estudo, pois verificou-se aumento gradativo da temperatura axilar aos 60 minutos no grupo submetido à posição vertical, sugerindo o aumento no valor dessa variável provavelmente devido à condução de calor da mãe ao filho. Apesar da hipotermia ter sido relatada por Lima et al. $11 \mathrm{em}$ uma taxa de 30 episódios de hipotermia leve/100 bebêsdias, esse evento não ocorreu no presente estudo. É possível que o intervalo de uma hora entre a observação de cada pré-termo em cada grupo, bem como os cuidados ambientais, possam ter minimizado esse efeito. A medida da temperatura da região axilar direita forneceu um resultado aproximado da temperatura central, sensível para o propósito do estudo, que não estava considerando hipertermia. Como a medida da temperatura central deve ser aferida por via retal em uma profundidade de $2 \mathrm{~cm}$, essa não foi utilizada considerando o possível risco de perfuração do reto ou cólon sigmóide descrito por Thilo e Rosemberg. ${ }^{9}$ Entretanto, Constantinou et al. 20 publicaram um estudo sobre a medida da temperatura axilar, antes e após a realização da Posição MãeCanguru, com bebês pré-termos de baixo peso observando temperatura corpórea estável nesses períodos. Também, Blaymore et al. 13 verificaram a estabilidade da temperatura axilar e retal e da frequiência respiratória observadas durante dez minutos da instituição dessa posição. Tais diferenças poderiam ser explicadas pela duração no tempo de aferição da temperatura no primeiro estudo (nos dez primeiros minutos) e as diferenças no desenho e do objetivo do segundo estudo citado. Ainda, deve ser notado que ambos os trabalhos procuraram demonstrar a segurança do método, salientando que a queda na temperatura axilar não havia ocorrido.

Não foram encontrados estudos indicando aumento da freqüência respiratória nos pré-termos submetidos à Posição Mãe-Canguru. Isto é compatível com os resultados encontrados neste trabalho, no qual não se observou aumento da freqüência respiratória no grupo que realizou o contato pele a pele. Sugere-se, assim, que a estabilidade na frequiência respiratória tenha ocorrido devido à diminuição de apnéias ou pausas respiratórias, enquanto o bebê per- 
maneceu na posição vertical, em contanto com o peito da mãe.

A estabilização da temperatura parece diminuir os episódios de apnéia, resultantes da imaturidade do centro respiratório e dos mecanismos de proteção que mantém a patência das vias aéreas, principalmente em recém-nascidos pré-termos. ${ }^{18,24}$ Estudos prévios demonstraram que a monitorização respiratória pode detectar alguns tipos de apnéia (obstrutiva) e deve, por essa razão, sempre ser combinada com a monitorização da freqüência cardíaca e da saturação de oxigênio. ${ }^{14,25}$ Portanto, este estudo realizou essa monitorização, em diferentes períodos ao longo de uma hora de observação da Posição Mãe-Canguru, no intuito de detectar possíveis alterações.

\section{Conclusão}

A pesquisa apresentou a aplicabilidade da Posição Mãe-Canguru como uma técnica segura para a práti- ca fisioterapêutica no tratamento de bebês semelhantes aos deste estudo. Tais resultados, apesar de serem superponíveis aos de estudos clínicos conduzidos em outros centros, poderiam ser melhor detalhados a partir de uma amostra com maior número de pacientes.

Assim, os pré-termos submetidos à Posição MãeCanguru, durante o período de uma hora, apresentaram aumento nos valores das respostas fisiológicas estudadas, em comparação com o mesmo período em que foram submetidos à posição elevada em decúbito ventral na incubadora, sendo essas diferenças estatisticamente significantes, exceto para a freqüência respiratória.

$\mathrm{Na}$ área da Fisioterapia, desenvolve-se a perspectiva de continuidade dessa pesquisa nas variantes de tempo de aplicação da Posição Canguru e da realização do Método Mãe-Canguru em sua totalidade, ampliando a inserção do profissional fisioterapeuta na área da neonatologia e estabelecendo sua participação nesse campo de atuação.

\section{Agradecimentos}

Aos médicos pediatras Paulo e Silvana Nader e aos médicos neonatologistas Daniel Chazan e Ivana Varella, pelo apoio e incentivo na realização deste trabalho, bem como às equipes médica e de enfermagem do Hospital da Criança Conceição e do Hospital Nossa Senhora da Conceição, pelo empenho, sugestões e contribuições durante a realização desse trabalho.

\section{Referências}

1. Feldman R, Eidelman A. Intervention programs for premature infants - How and do they affect development? Clin Perinatol 1998; 25: 613-26.

2. Fleisher BC, Vandenberg K, Constantinou J, Heller C, Benitz WE, Johnson A, Rosenthal A, Stevenson DK. Individualized developmental care for very-low birthweight premature infants. Clin Pediatr 1995; 34: 523-9.

3. Glass P. O recém-nascido vulnerável e o ambiente na unidade de tratamento intensivo neonatal. In: Avery GB, Fletcher MA, MacDonald M. Neonatologia: fisiopatologia e tratamento do recém-nascido. 4. ed. Belo Horizonte: Medsi; 1999. p. 79-96.

4. Charpak N, Calume ZF, Hamel A. O Método Mãe-Canguru: pais e familiares dos bebês pré-termos podem substituir as incubadoras. Rio de Janeiro: McGraw-Hill; 1999.

5. Charpak N, Ruiz-Pelaez JG, Figuereoa Z, Charpak Y. Kangaroo mother care versus traditional care for newborn infants $£$ to 2000 grams: a randomized, controlled trial. Pediatrics 1997; 100: 682-8.

6. Bauer K, Uhrig C, Sperling KP, Wiland C, Versmold HT. Body temperatures and oxygen consuption during skinto-skin (kangaroo) care in stable preterm infants weighing less than 1500 grams. J Pediatr 1997; 130: 240-4.

7. Christensson K, Bhat GJ, Amadi BC, Eriksson B, Höjer B. 
Randomised study of skin-to-skin versus incubator care for rewarming low risk hypothermic neonates. Lancet 1998; 352: 1115.

8. Bohnhorst B, Heyne T, Peter CS, Poets CF. Skin-to-skin (kangaroo) care, respiratory control, and thermoregulation. J Pediatr 2001; 138: 193-7.

9. Thilo EH, Rosemberg AA. The newborn infant. In: Hay WWJr, Hayward AR, Levin MJ, Current pediatric diagnosis \& treatment. 14th ed. Stamford: Appleton \& Lange; 1999.

10. Carvalho MR. Método Mãe-Canguru de atenção ao prematuro. Rio de Janeiro: BNDES; 2001. p. 9-47.

11. Lima G, Quintero-Romero S, Cattaneo A Feasibility, acceptability and cost of Kangaroo mother care in Recife, Brazil. Ann Trop Paediatr 2000; 20: 22-6.

12. Bauer K, Sontheimer D, Fischer C, Linderkamp O. Metabolic rate and energy balance in very low birth weigh infants during kangaroo holding by their mothers and fathers. J Pediatr 1996; 129: 608-11.

13. Blaymore BJA, Ferguson AE, Morales Y, Liebling JAMS Archer D, Oh W, Vohr BR. Comparison of skin-to-skin contact with standard contact in low birth weight infants who are breast-fed. Arch Pediatr Adolesc Med 1996; 150: $1265-9$.

14. Sontheimer D, Fischer CB, Scheffer F, Kaempf D, Linderkamp O. Pitfalls in respiratory monitoring of premature infants during kangaroo care. Arch Dis Child Fetal Neonat 1995; 72: 115-7.

15. Crane LD. Fisioterapia para o neonato com doença respiratória. In: Irwin S, Tecklin JS. Fisioterapia cardiopulmonar. 2. ed. São Paulo: Manole; 1999. p. 381-407.

16. Martin RJ, Merrel N, Rubin D, Fanaroff A. Effect of supine and prone positions on arterial oxygen tension in the preterm infant. Pediatrics 1979; 63: 528-531.
17. Kornecki A, Frndova H, Coates AL, Shemie SD. A randomized trial of prolonged prone positioning in children with acute respiratory failure. Chest 2001; 119: 211-8

18. Bohn D. Lung salvage and protection ventilatory techniques. Pediatr Clin North Am 2001; 48: 553-72.

19. Jardim JRB, Filho SPC. Fisiologia pulmonar. In: Kopelman BI, Miyoshi MH, Guinsburg R. Distúrbios respiratórios no período neonatal. São Paulo: Atheneu; 1998. p. 1543.

20. Constantinou JCMA, Adamson-Macedo EN, Stevenson DK, Majid M, Fleisher BE. Effects of skin-to-skin holding general movements of preterm infants. Clin Pediatr 1999; 38: 467-71.

21. Kollef MH. Critical care parameters and formulas. In: Haya SN, Flood K, Paranjothi S. The Washington manual of medical therapeutics. 30th ed. Philadelphia: Lippincott Williams \& Wilkins; 2001. p. 628-9.

22. Ludington-Hoe SM, Hadeed GC, Anderson GC. Physiologic responses to skin-to-skin contact in hospitalized premature infants. J Perinatol 1991; 11: 19-24.

23. Guyton AC, Hall JE. O músculo cardíaco: o coração como uma bomba. In: Guyton AC, Hall JE. Tratado de fisiologia médica. 9. ed. Rio de Janeiro: Guanabara Koogan; 1997. p. 87-108.

24. Hey E. Termorregulação. In: Avery GB, Fletcher MA, MacDonald M. Neonatologia: fisiopatologia e tratamento do recém-nascido. 4. ed. Belo Horizonte: MEDSI; 1999. p. 360-8.

25. Warburton D, Stark AR, Taeusch HW. Apnea monitor failure in infants with upper airway obstruction. Pediatrics 1977; 60: 742-74. 